\title{
Laser Treatment Based on Minimal Intervention - Report of Cases
}

\author{
NA Nomann ${ }^{1}$, MR Hasan $^{2}$, AB Dithi $^{3}, \mathrm{~K} \mathrm{Endo}^{4}$, T Saito $^{5}$
}

\begin{abstract}
Since the development of the ruby laser by Maiman in 1960, many lasers are available today for clinical application in dentistry, based on minimally invasive (MI) dental treatment in routine clinical procedures including dental cavity preparation, removal of dental calculus, caries prevention, and treatment of dentin hypersensitivity, anti-inflammation for soft tissues, pain relief, surgical operations, and hemostasis. Dental treatment using a laser is a minimally invasive, painless treatment with a low burden on the patient, and may therefore be seen as a treatment that complies with the MI concept. However, safe use of lasers must be the underlying goal of proposed or future laser therapy. To ensure that safe, effective MI treatment is practiced when using lasers, it is essential to have a full understanding of their advantages and disadvantages before using them.
\end{abstract}

Key words: Laser, Minimal Intervention, Caries Removal, Removal of Melanin Pigmentation.

\section{Introduction}

There has recently been a rapid spread of treatments using lasers in the field of dentistry 1,2 . Lasers are now widely used in minimally invasive (MI) dental treatment in routine clinical procedures including dental cavity preparation $^{3}$, removal of dental calculus ${ }^{4}$, caries

1. Dr. Nahid A1 Nomann BDS, Doctoral Fellow, Division of Clinical Cariology and Endodontology,, Department of Oral Rehabilitation, School of Dentistry, Health Sciences University of Hokkaido, , Ishikari-Tobetsu, Hokkaido, Japan.

2. Dr. Md. Riasat Hasan BDS, MPH, FRSPH (UK), Doctoral Fellow, Division of Clinical Cariology and Endodontology, Department of Oral Rehabilitation, School of Dentistry, Health Sciences University of Hokkaido, Ishikari-Tobetsu, Hokkaido, Japan.

3. Dr. Akashlynn Badruddoza Dithi BDS, Doctoral Fellow, Division of Biomaterials and Bioengineering, Department of Oral Rehabilitation, School of Dentistry,

Health Sciences University of Hokkaido,

Ishikari-Tobetsu, Hokkaido, Japan.

4. Prof. Kazuhiko Endo $\mathrm{PhD}$, Division of Biomaterials and Bioengineering, Department of Oral Rehabilitation, School of Dentistry, Health Sciences University of Hokkaido, Ishikari-Tobetsu, Hokkaido, Japan.

5. Prof. Dr. Takashi Saito DDS, PhD, Division of Clinical Cariology and Endodontology, Department of Oral Rehabilitation, School of Dentistry, Health Sciences University of Hokkaido, Ishikari-Tobetsu, Hokkaido, Japan.

Address of Correspondence:

Prof. Dr. Takashi Saito DDS, PhD, Division of Clinical Cariology and Endodontology, Department of Oral Rehabilitation, School of Dentistry, Health Sciences University of Hokkaido, Ishikari-Tobetsu, Hokkaido, Japan., E-mail: t-saito@hoku-iryo-u.ac.jp prevention $^{5}$, treatment of dentin hypersensitivity, anti-inflammation for soft tissues ${ }^{7}$, pain relief, surgical operations ${ }^{8}$, and hemostasis.

Laser is an acronym for "light amplification by stimulated emission of radiation"1. Einstein put forward his theory of stimulated emission in 1917, and Maiman successfully generated a ruby laser beam in 1960. Since then, many different types of lasers have been developed. A laser beam is light, which is a type of electromagnetic wave and has the characteristics of coherence (wavelength and phase are uniform), monochromacy, directionality (the beam is straight without spreading out), condensability, and high output capability. The laser can have thermal and non-thermal (photochemical) effects on an irradiated body. Non-thermal effects may be further classified as photic stimulation, photochemical effects, photo-dissociation effects, and photo impact wave effects. These effects depend mainly on the energy and the wavelength of the laser. For example, lasers with wavelengths in the infrared region, such as the $\mathrm{CO}_{2}$ laser $(10.6 \mu \mathrm{m})$, Er:YAG laser $(2.94 \mu \mathrm{m})$, or Nd:YAG laser $(1.06 \mu \mathrm{m})$, mainly produce thermal effects. The sudden rise in temperature of the exposed area causes effects such as ablation or solidification in tissues such as dental hard tissue. Lasers with wavelengths in the ultraviolet region, such as the excimer laser $(0.248 \mu \mathrm{m})$, have non-thermal effects and can be used in applications such as pain relief. Lasers with wavelengths in the visible ray region, such as the He-Ne laser $(0.633 \mu \mathrm{m})$ and diode laser $(0.655-0.900 \mu \mathrm{m})^{9}$, have both effects and are used in applications such as pain relief and photodynamic therapy (PDT). Diode lasers are also used at high power for surgical operations and solidification.

$\mathrm{CO}_{2}$ and Er:YAG lasers show surface absorbency, whereas Nd:YAG, diode, and He-Ne lasers are able to penetrate tissues. These characteristics need to be taken into account in the use of lasers. 
Here, we used routinely encountered cases to explain approaches to operative and cosmetic dental treatment with lasers based on the concept of minimal invasion, which is our area of specialty, and examined the significance of such approaches.

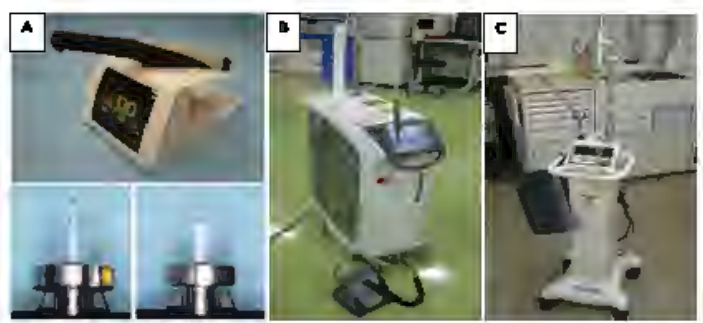

Figure 1 - LASERS

A: DIAGNOdent Pen (KaVo), B: Erwin AdvErL EvoMorita), C: Super Pulse Laser LX-20SPLUXAR

Case 1 (Figs. 1A, 1B, 2)

The patient was a 23-year-old man who attended the clinic for a regular checkup. Coloration was found in the pit and fissure of the occlusal surface of the maxillary left second molar. There was no symptom in particular (Fig. 2). As there were no clear findings from X-nay examination, an examination for caries was carried out by the laser fluorescence method ${ }^{10}$. The resulting diagnosis was that restarative treatment was needed from the central pit along the buccal groove, and preventative treatment was needed in the distal pit. Ablation of dental hard tissue was carried out under running water by using the Erwin AdvErL Evo Er:YAG laser (Morita Corp.) ${ }^{11}$ at 10 pps and $150 \mathrm{~mJ}$, after which restarative treatment was carried out by using the Bond Force one-step bonding system and the Estelite Flow Quick/Estelite $\mathbf{\Sigma}$ Quick system (Tokuyama Dental Corp.). Laser fluorescence is a method for examining dental caries by irradiating healthy and carious dental hard tissue with a diode laser $(655 \mathrm{~nm})$ and measuring the difference in intensity of the fluorescence reflection. Laser fluorescence can he used effectively and non-invasively for follow-up observations and for determining dental hard tissue that needs preventative or restorative treatment, and it is a highly accurate and reproducible method of examination. Also, as Er:YAG lasers show strong absorption in water, they react with the bydration sphere of a hydroxyapatite surface to produce heat, and the tiny blasts (ablation) from this heat together with the photo impact effect of the pulse wave (photo-mechanical process) can cut tooth tissue. The smear layer on the surface of the dentine produced when cutting with a rotary drilling device does not form when using lasers. However, a denatured layer forms, which works unfavorably for bonding, so this layer needs to be removed with a spoon excavator before bonding.

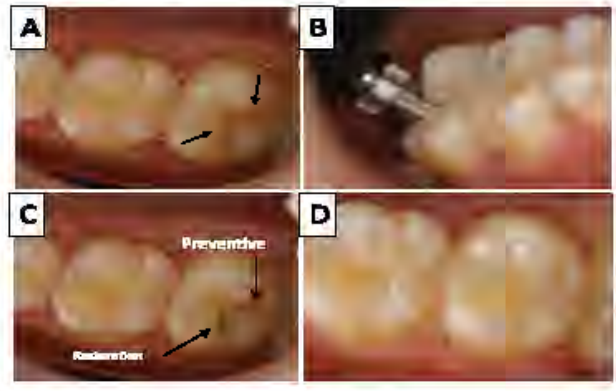

Figure 2 Case 1

A: First visit (\#27)

fluorescence method

C: Result of diagnosis D: After treatment(composite resin restaration in buccal fissure after caries removal using Er:YAG lager and preventive care in distal pit)

\section{Cave 2 (Fig. 1C, Fig. 3)}

The patient was a 24-year-old woman, who attended the clinic with the main complaint of poor esthetic appearance of the gums. The woman was a smoker (Fig. 3). The patient was diagnosed with melanin pigmentation, and after she was given guidance in giving up smoking, the areas of gum discoloration in the upper and lower jaw were removed under surface anesthesia by irradiation with a Super Pulse Laser LX-20SP CO, laser (LUXAR) $^{12}$ using a ceramic tip at $4 \mathrm{~W}$ and a continuous wave. At around 3 postoperative days, she experienced stinging pain on the surface of the gums, but no other problems were found. The same treatment was carried out after an interval of 10-14 days, and as the patient was satisfied, the treatment was brought to completion. $\mathrm{CO}_{2}$ lasers have thermal effects on tissues, and the sudden rise in temperature of the irmadiated aree causes ablation and solidification. Because the gums of the front teeth are thin, the effects of thermal stimulation on the surrounding tissues need to he taken carefully into account.

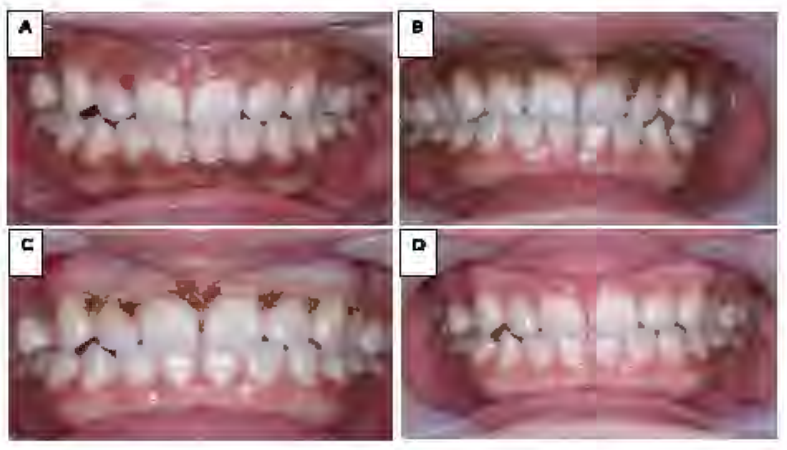

Figure 3 Case 2

A: Finst visit

B: Removal of melanin pigmentation with $\mathrm{CO}_{2}$ laser

C: Re-irradiation after 14 days

D: After 6 months 


\section{Discussion}

When used for caries treatment, lasers have the huge advantage that they mitigate the fear of dental treatment because they have none of the unpleasant noise and vibration of rotary drilling devices. Also, as anesthesia is not normally needed, lasers have the advantage that they can be used without anxiety for patients suffering systemic disorders such as high blood pressure. Lasers can be used to carry out caries treatment easily on patients with small mouths such as young children, and because little heat is produced while cutting, treatment can be carried out while the tooth pulp is kept in check with a small amount of running water. Also, lasers have advantages when used on soft tissues in that they suppress bleeding, have sterilizing and detoxification effects, increase the rate of healing, and cause little postoperative pain. The disadvantages include the time it takes to cut through tooth tissue, roughness of the cut surface, the high cost of equipment, and the possible risks if the safety management is not perfect. In particular, to ensure safety when lasers are used, laser equipment must, of course, be inspected before use and properly maintained, and staff must have safety training. In addition, special protective glasses must be worn to prevent any damage to the retina, cornea, or crystalline lens, and care must be taken to ensure as far as possible that the skin is not exposed to the laser.

\section{Conclusion}

As we have noted here, dental treatment using a laser is a minimally invasive, painless treatment with a low burden on the patient, and may therefore be seen as a treatment that complies with the MI concept. To ensure that safe, effective MI treatment is practiced when using lasers, it is essential to have a full understanding of their advantages and disadvantages before using them.

Lasers have been in use in medicine for no more than half a century. During this time, the development of lasers and the accumulation of evidence-based medicine using them have been astonishing. For example, there is great interest these days in $\mathrm{CAD} / \mathrm{CAM}$ and 3D printers using lasers.

There have also been advances in the analyses of biological effects and mechanisms and in the development of new methods of diagnosis and treatment using lasers. Lasers are likely to develop further as the "dream light" for next-generation dental treatments.

\section{References}

1. Wigdor HA, Walsh JT Jr, Featherstone JD, Visuri SR, Fried D, Waldvogel JL. Lasers in dentistry. Lasers Surg Med. 1995;16(2):103-133.

2. Coluzzi DJ. Lasers in dentistry. Compend Contin Educ Dent. 2005; 26(6ASuppl):429-435.

3. de Azevedo CS, Garbui BU, E Silva CM, Lorenzetti MR Simionato, de Freitas AZ, Matos AB. Obtaining Artifcially Caries-affected Dentin for in vitro Studies. J Contemp Dent Pract. 2014;15(1):12-19.

4. Schoenly JE, Seka W, Rechmann P. Pulsed laser ablation of dental calculus in the near ultraviolet. $\mathrm{J}$ Biomed Opt. 2014;19(2):028003.

5. Moshonov J, Stabholz A, Zyskind D, Sharlin E, Peretz B. Acid-etched and erbium:yttrium aluminium garnet laser-treated enamel for fissure sealants: a comparison of microleakage. Int J Paediatr Dent. 2005;15(3):205-209.

6. Bader J, Balevi B, Farsai P, Flores-Mir C, Gunsolley J, Matthews D, Vig K, Zahrowski J. Lasers may reduce pain arising from dentin hypersensitivity. J Am Dent Assoc. 2014;145(4):e1-2.

7. Neiburger EJ. The effect of low-power lasers on intraoral wound healing. N Y State Dent J. 1995;61(3):40-43.

8. Jarjoura K. Soft tissue lasers. Am J Orthod Dentofacial Orthop. 2005;127(5):527-528.

9.Slot DE, Jorritsma $\mathrm{KH}$, Cobb CM, Van der Weijden FA. The effect of the thermal diode laser (wavelength $808-980 \mathrm{~nm}$ ) in non-surgical periodontal therapy: a systematic review and meta-analysis. J Clin Periodontol. 2014;41(7):681-692.

10. Haak R, Wicht MJ. Caries detection and quantification with DIAGNOdent: prospects for occlusal and root caries? Int $\mathrm{J}$ Comput Dent. 2004;7(4):347-358.

11. Fornaini C. Er:YAG and adhesion in conservative dentistry : clinical overview. Laser Ther. 2013;22(1):31-35.

12. Esen E, Haytac MC, Oz IA, Erdoğan O, Karsli ED. Gingival melanin pigmentation and its treatment with the $\mathrm{CO}_{2}$ laser. Oral Surg Oral Med Oral Pathol Oral Radiol Endod. 2004;98(5):522-527. 